\title{
Review of: "Spatial organization of nuclear pores in Xaenopus laevis oocytes"
}

\author{
Tristan Aguero ${ }^{1}$ \\ 1 Universidad Nacional de Tucumán
}

Potential competing interests: The author(s) declared that no potential competing interests exist.

Review of: Spatial organization of nuclear pores in Xaenopus laevis oocytes

This paper aims to study how nuclear pores are arranged on the nuclear surface. They used superresolution images of nuclei surfaces of Xenopus laevis oocytes at different stages of maturation for the analysis. They characterize the arrangement of nuclear pore using commonly used tools to study atomic structural and topological features of ordinary matter, such as numerical simulations of octagonal clusters to resemble typical pore shapes. Based on statistical analysis of the geometrical arrangement they concluded that nuclear pores arrangement does not depend on pore-pore interaction, but rather on their octagonal geometry, suggesting that this conformation plays a central role in nuclear pores organization.

\section{Title, abstract and introduction:}

1. The first thing that caugth my attention was the misspelled word Xenopus, even in the tittle.

2. The second one, and most notorious without a doubt was the poor English language used to write this paper. I understand the authors are not native English speakers but there are many options to improve the language of a manuscript before sending it for publication. This paper is not easy to read since English is not fluently written. There are several structural and grammatical mistakes, for example, and just to mention a few, inaccurate verb tenses, misuse of adverbs instead of adjectives, some statements were redundant other confusing, etc.

3. Unfortunately, the poor English used to write this paper makes it really hard to read and follow.

\section{Results:}

1. All the analyzed parameters were correct, well-selected, and well-founded.

2. I found each experimental approach was designed without a fully explanation of why they are using the selected parameter for the analysis. It would be great if the authors used a biological explanation to fundament why an approach or an experiment was chosen. Explain what is the biological rationale behind the experiments, or what is the goal they want to pursuit in each approach. I think improving this manner of explanation will definitely ameliorate the global understanding of this research.

3. A regular reader in the molecular, cell or developmental biology field has no background in physics or 
mathematics therefore it is difficult to follow a paper that used several formulae to explain a biological process, like this paper, so I suggest to try to present a biological sense or an explanation of why they decided to do one experiment, what biological question they want to answer doing these experiments.

4. It would be great if the authors propose a question they want to answer or explain the problem they want to solve and then continue writing each result section. Set the rationale first, why they are going to do an experiment, and then tell the experiment they did and explain the result. This is the correct manner to writhe the result section in a paper. In this case the authors do not follow this rule and directly describe the experiment they did without the rationale behind that explain why they did it.

\section{Discussion and conclusion:}

1. Discussion has to be more analytical. It needs further contrast with other related publications or with the current knowledge in the topic, explaining the advantages of the findings presented in this work or how these findings will contribute to the knowledge in the field.

2. Conclusion is poorly described. Confusing actually. Please rewrite as clear and precise as possible since this short part of the publication is fundamental to present the final idea to the reader.

As a final remark, the finding present in this paper are interesting in the aim to demonstrate the behavior of nuclear pores at different biological situations, contrasting what happen in a less mature and more transcriptional active oocyte versus a more mature and less active one. But there are several aspects to improve to reach a well-written, well-founded, precise and complete paper ready to be published. 